\title{
Correction to: Crosstalk between cancer cells and endothelial cells: implications for tumor progression and intervention
}

\author{
Hyojeong Choi ${ }^{1} \cdot$ Aree Moon $^{1}$ (D)
}

Published online: 29 August 2018

(C) The Pharmaceutical Society of Korea 2018

\section{Correction to: Arch. Pharm. Res. (2018) 41:711-724 https://doi.org/10.1007/s12272-018-1051-1}

Unfortunately, there is an error in the original version of the article. The original published article contains an incomplete 'Acknowledgements' section. Please find the full 'Acknowledgements' section below.

\begin{abstract}
Acknowledgements This study was supported by the Duksung Women's University Research Grant 2017 and the Priority Research Centers Program (No. 2016R1A6A1A03007648) of the National Research Foundation (NRF) funded by the Korean Government.
\end{abstract}

The original article can be found online at https:// doi.org/10.1007/s12272-018-1051-1.

Aree Moon

armoon@duksung.ac.kr

1 Duksung Innovative Drug Center, College of Pharmacy,

Duksung Women's University, Seoul 01369, Korea 